\title{
Zu den Auswirkungen von Hartz IV auf den Arbeitsmarkt - Fakten und Fragen
}

Effects of Hartz IV on the Labour Market - Facts and Questions

Peter Bartelheimer*, Volker Baethge-Kinsky*, Alexandra Wagner ${ }^{\circ}$

\section{"Hartz-Gesetze" - eine strategische Weichenstellung}

Mit Jahresbeginn 2005 ist mit dem SGB II auch das letzte der vier »Hartz-Gesetze« zur Neuausrichtung der Arbeitsmarktpolitik des Bundes in Kraft getreten, mit denen die Bundesregierung im Rahmen der »Agenda 20IO« die Empfehlungen der Hartz-Kommission »Moderne Dienstleistungen am Arbeitsmarkt « umsetzen will. Diese Gesetze betreffen nicht allein Erwerbslose. Mit ihnen sind Richtungsentscheidungen für die künftige soziale Absicherung von Arbeitsmarktrisiken im deutschen Sozialstaat gefallen, die als größtes sozialpolitisches Experiment seit der Herstellung der deutschen Einheit bezeichnet werden können. Abstimmungsprobleme zwischen bestimmten Formen der Flexibilität am Arbeitsmarkt und dem bundesdeutschen Modell sozialer Sicherung, also Probleme des "Mismatch zwischen dem institutionellen System des Arbeitsmarktes einerseits und demjenigen des Sozialleistungssystems [andererseits] « (Klammer / Tillmann 2002: I6 f.), tragen seit Jahrzehnten zu beruflichsozialer Gefährdung, materieller Armut und sozialer Ausgrenzung bei. Im Folgenden soll kurz zusammengefasst werden, wie die »Hartz-Gesetze» die Grundlage für solche Diskussionen verändern und warum sie die Möglichkeiten, Flexicurity-Lösungen für Risiken unsicherer Erwerbsbeteilung weiter zu verfolgen, entscheidend einschränken.

Mit den »Hartz-Gesetzen « wurden zwei Richtungsentscheidungen durchgesetzt, die es rechtfertigen, von einer grundsätzlichen Neuausrichtung der Arbeitsmarktpolitik und der Arbeitsförderung zu sprechen.

Erstens zielen die »Hartz-Gesetze» darauf ab, den Bestand an gemeldeter, d.h. sozialstaatlich wahrgenommener Arbeitslosigkeit unabhängig vom makroökonomischen Umfeld, also auch ohne Beschäftigungszuwächse, durch Beeinflussung des individuellen Arbeitsmarktverhaltens Arbeitsuchender zu verringern. Der Übergang von einer aktiven zu einer "aktivierenden « Arbeitsmarktpolitik (vgl. Koch/Walwei 2005, Konle-Seidl 2005) soll den Bestand an gemeldeter Arbeitslosigkeit durch Verkürzung der individuellen Dauer von Arbeitslosigkeitsphasen senken. Vorrangig soll dies durch raschere Besetzung offener Stellen aufgrund besserer Vermittlung und erhöhter Konzessionsbereitschaft der Arbeitsuchenden

* SOFI, Soziologisches Forschungsinstitut an der Georg-August-Universität Göttingen.

- $\quad$ FIA Forschungsteam Internationaler Arbeitsmarkt GmbH, Berlin.

- Die Autor|inn|en bearbeiten aktuell das von der Hans-Böckler-Stiftung und der Otto-BrennerStiftung geförderte Projekt »Monitor Arbeitsmarktpolitik. Ein Projekt zur wissenschaftsgestützten Begleitung der Umsetzung der neuen Arbeitsmarktgesetze (Hartz I bis IV)«. 
erreicht werden, aber auch die Abmeldung sogenannter »unechter« Arbeitsloser aus der Statistik dient diesem Ziel. Die Entscheidung, Arbeitslosigkeit nur noch auf der Mikroebene individuellen Verhaltens zu bearbeiten, individualisiert das gesellschaftliche Problem der Unterbeschäftigung radikal. Bearbeitet wird nicht mehr eine gesamtwirtschaftliche Beschäftigungslücke. Vielmehr sollen Defizite persönlicher Beschäftigungsfähigkeit durch Maßnahmen der Eingliederung in ein Beschäftigungssystem ausgeglichen werden, das selbst kaum noch Gegenstand arbeitsmarktpolitischer Intervention ist. Dieser Politikwechsel, der bereits 1998 mit der Neufassung des Zielkatalogs für Arbeitsförderung im SGB III begann und 2002 mit dem Job-AQTIV-Gesetz weiter vorangetrieben wurde, ist mit der Fürsorgelogik des SGB II endgültig vollzogen. Er geht einher mit einer skeptischen KostenNutzen-Einschätzung klassischer Instrumente der Arbeitsförderung, insbesondere direkt Beschäftigung schaffender Maßnahmen, aber auch teurer Maßnahmen der Aus- und Weiterbildung. Diese Skepsis beruht zum Teil darauf, dass der Nutzen von Arbeitsförderung überwiegend an engen Kriterien der unmittelbaren Wiedereingliederung in ungeförderte Beschäftigung gemessen wird und indirekte sowie langfristige Effekte kaum betrachtet werden.

Zweitens wird mit der Zusammenlegung von Arbeitslosenhilfe und Sozialhilfe zum SGB II, das sich im wesentlichen am materiellen und sozialrechtlichen Niveau der Sozialhilfe orientiert bzw. dieses noch unterschreitet, eine bereits wirksame Tendenz zur Polarisierung sozialer Schutzrechte am Arbeitsmarkt sozialrechtlich festgeschrieben. "Hartz IV « schafft nicht ein System für alle Arbeitsuchenden, sondern stellt dem nun enger begrenzten Bereich der Arbeitslosenversicherung (SGB III) ein nach dem Fürsorgeprinzip neu gestaltetes Leistungssystem (SGB II) gegenüber, welches fast jede Arbeit für zumutbar erklärt, den Leistungsbezug von der Einhaltung von persönlichen Verhaltensanforderungen abhängig macht (die vom Leistungsträger im Rahmen verpflichtender Eingliederungsvereinbarungen einseitig festgelegt werden können) und bei Nichteinhaltung Sanktionsmöglichkeiten bis hin zur vollständigen Streichung der Leistungen zum Lebensunterhalt vorsieht. Diese „Grundsicherung für Arbeitsuchende« steht der Erwerbslosenfürsorge der Weimarer Republik näher als dem - in das SGB XII überführten - Bundessozialhilfegesetz und hat mit in den I990er Jahren diskutierten Reformkonzepten der Grundsicherung nur mehr den Namen gemeinsam. Die Trennung der arbeitsmarktnahen sozialen Sicherung in zwei gegeneinander abgegrenzte, in der konkreten Umsetzung oft auch räumlich getrennte Regelungsbereiche erschwert die Verwirklichung zweier sozialer Rechte: des Rechts auf wirtschaftliche Sicherung bei Arbeitslosigkeit ( $\$_{3}$ SGB I) und auf grundsichernde Sozialleistungen (\$9 SGB I) einerseits und des Rechts auf Bildungs- und Arbeitsförderung ( $\$_{3}$ SGB I) andererseits.

In der materiellen Existenzsicherung werden mit dem SGB II sozialstaatliche Spaltungslinien nach unten und nach oben vertieft. Für ihren Lebensunterhalt erhalten bedürftige Erwerbsfähige, die keinen Anspruch auf Versicherungsleistungen haben, die steuerfinanzierte Fürsorgeleistung Arbeitslosengeld II (Alg II), ihre nicht erwerbsfähigen Familienangehörigen Sozialgeld. Da Arbeitslosenhilfe und Sozialhilfe auf dem Leistungsniveau der Sozialhilfe zusammengeführt wurden, bedeutet der Übergang von Lohnersatzleistungen, die sich noch am früheren Erwerbseinkommen orientierten, auf die Sicherung des Existenzminimums (nach einer scharfen Bedürftigkeitsprüfung, die Partnereinkommen und Erspar- 
nisse bis auf einen geringen Rest sowie die Wohnkosten einbezieht) einen tiefen materiellen Abstieg. Nur nach Bezug von Arbeitslosengeld I wird dieser Abstieg durch einen befristeten Zuschlag noch vorübergehend abgefedert.

Die »Aussteuerung « aus der Arbeitslosenversicherung wegen fehlender oder erschöpfter Versicherungsansprüche führt nicht nur zu kurzfristigen Einkommenseinbußen, sondern wird auch in biografischer Perspektive riskant: Wer einmal in den Bezug von Alg II übergegangen ist, hat geringere Chancen als früher, wieder aus der Fürsorge in das Versicherungssystem aufzusteigen; Ansprüche hierauf können nur noch durch eine ausreichend lange versicherungspflichtige Erwerbstätigkeit am »ersten Arbeitsmarkt« erworben werden. Arbeitsbeschaffungs-, Qualifizierungsmaßnahmen und Arbeitsgelegenheiten mit Mehraufwandsentschädigung (»Ein-Euro-Jobs») ermöglichen dagegen keine Rückkehr in die Arbeitslosenversicherung mehr.

Leistungsansprüche nach SGB II für bedürftige Erwerbslose sowie Geringverdiener|innen ohne Versicherungsansprüche sind noch weniger rechtssicher als bisherige Sozialhilfeleistungen, da an unbestimmte Verhaltensanforderungen und Gegenleistungen (»Fordern und Fördern«) gebunden, und sie lassen sich leichter stigmatisieren. Für Erwerbsfähige im Zuständigkeitsbereich des SGB II gilt das Bedarfsdeckungsprinzip der Sozialhilfe nicht mehr. Soziokulturelle Mindestnormen der Teilhabe sind für sie weniger verbindlich als für Leistungsberechtigte nach SGB XII, da die Geldleistung zeitweise abgesenkt werden oder ganz wegfallen kann. Bei der Bemessung der Leistungshöhe und bei der Gestaltung von Anreizen (Anrechnung von Erwerbseinkommen) und Sanktionen (Absenkung und Wegfall von Leistungen) kann sich daher das ökonomische Interesse an einer Senkung des Anspruchslohns im Niedriglohnsektor und an einer Subventionierung von Niedrigeinkommen durch lohnergänzende Fürsorgeleistungen ungehindert durchsetzen.

Bezieher|inne|n von Alg II ist die Aufnahme jeder angebotenen Arbeit und die Teilnahme an jeder angebotenen Maßnahme zumutbar ( $\$$ Io SGB II). Einen positiven Anspruch auf Integrationsmaßnahmen gibt es hingegen nicht. »Leistungen zur Eingliederung in Arbeit« sind grundsätzlich Kann-Leistungen (\$ 3 Abs. I SGB II). Zwar können viele Instrumente der Arbeitsförderung nach SGB III grundsätzlich auch Bezieher|inne|n der Grundsicherung für Arbeitsuchende angeboten werden ( $\$$ I6 Abs. I SGB II). Der Aussteuerungsbetrag aber, den die Bundesagentur für Arbeit (BA) beim Übergang von Versicherten zum Alg II zu zahlen hat, setzt für die Arbeitsagenturen einen fiskalischen Anreiz, von dieser Kann-Bestimmung keinen Gebrauch zu machen und ihre Fördermittel vorrangig für Arbeitslosengeldbezieher|innen einzusetzen. Im Großen und Ganzen beschränkt sich damit die Arbeitsförderung im Fürsorgebereich auf zwei besondere Instrumente: auf öffentlichrechtliche Arbeitsgelegenheiten, die kein Beschäftigungsverhältnis begründen, mit einer Mehraufwandsentschädigung entgolten werden (»Ein-Euro-Jobs«, vgl. \$ I6 Abs. 3 SGB 2) und kaum zur Eingliederung in den Arbeitsmarkt beitragen dürften ${ }^{\mathrm{I}}$, sowie das Einstiegsgeld

I Die Arbeitsgelegenheiten mit Mehraufwandsentschädigung machten im Oktober 2005 mit 289.358 bzw. 79,7 Prozent den übergroßen Anteil aller Beschäftigung schaffenden Maßnahmen aus. Demgegenüber hatten Arbeitsbeschaffungsmaßnahmen (ABM) einen Anteil von 13,3 Prozent 
( $\$ 29$ SGB II). Dass Arbeitsuchende im Geltungsbereich des SGB II nur noch Objekte der Fürsorge sind, zeigt sich im Konstrukt der verpflichtenden Eingliederungsvereinbarung ( $\$$ I5 SGB II), die das Verhalten der Leistungsberechtigten weitgehend festlegt. Sie ist nur der äußeren Form nach eine freie Vereinbarung, da sie im Konfliktfall durch einen Verwaltungsakt ersetzt werden kann, und da Ablehnung oder Nichteinhaltung die regelhafte Absenkung des Alg II (bis hin zum Wegfall) zur Folge haben (vgl. Henke 2004).

Lohnabhängige in Beschäftigungsverhältnissen, die annähernd der Schutznorm des Normalarbeitsverhältnisses entsprechen (einschließlich der Beschäftigten in versicherungspflichtiger Teilzeit), und die Leistungen der Arbeitslosenversicherung nur in relativ kurzen Phasen der Sucharbeitslosigkeit wahrnehmen, sind von der Neuausrichtung der Arbeitsmarktpolitik nur in wenigen Bereichen direkt betroffen: etwa durch die Lockerung des Kündigungsschutzes, durch die erleichterte Befristung bei älteren Arbeitnehmer|inne|n und durch die Verpflichtung zur frühzeitigen Arbeitslosmeldung. In diesem Arbeitsmarktsegment relativ stabiler Beschäftigung, in dem die große Mehrheit der abhängig beschäftigten westdeutschen Männer arbeitet, wird vor allem die Verkürzung der Anspruchsdauer auf Arbeitslosengeld (auf maximal zwölf Monate bzw. I8 Monate für Ältere ab 55 Jahren) als erhöhtes soziales Risiko wahrgenommen.

Unmittelbar von den Neuregelungen betroffen ist dagegen ein Arbeitsmarktsegment von erheblichem Umfang, zu dem nicht nur Erwerbslose, sondern auch Beschäftigte zählen: eine breite Zone flexibler, nicht standardisierter und im deutschen Sozialversicherungssystem so nicht abgesicherter Erwerbsbeteiligung, für die niedrige Einkommen, Wechsel von Beschäftigung, Maßnahmen der Arbeitsförderung, Bezugszeiten von Lohnersatzleistungen und leistungslose Nichterwerbszeiten sowie Kombinationen von Erwerbseinkommen und lohnergänzenden Sozialtransfers typisch sind, und in der unbefristete, sozialversicherte Beschäftigung entsprechend dem Normalarbeitsverhältnis die Ausnahme bildet. Dieses Segment, dem in Westdeutschland vielleicht ein Zehntel der Erwerbspersonen zugerechnet werden kann, umfasst in Ostdeutschland etwa ein Drittel der Erwerbsbevölkerung. In den neuen Bundesländern stellen die Neuregelungen zudem Muster sozialstaatlich geförderter "sekundärer « Arbeitsmarktintegration in Frage, die in den I990er Jahren unter den besonderen Bedingungen der ostdeutschen Beschäftigungskrise entstanden und bislang dazu beitrugen, einen erheblichen Teil der Erwerbsbevölkerung bei anhaltender Unterbeschäftigung im Erwerbssystem zu halten (vgl. Land 2003, Alda et al. 2004a). Über subjektive Unsicherheitserfahrungen, Verdrängungseffekte und Lohneffekte wirkt die Neuausrichtung der Arbeitsmarktpolitik in beiden Landesteilen auch auf die Zone stabiler Beschäftigung zurück, die Flexibilisierung bisher vor allem in ihren unternehmensinternen Formen und bei relativer sozialer Sicherheit erfährt.

und Arbeitsgelegenheiten in der Entgeltvariante (Sozialversicherungspflicht ohne Arbeitslosenversicherung) einen Anteil von 3,7 Prozent. 


\section{Arbeitsmarktstatistik und erste Daten zu den Auswirkungen von Hartz IV}

Die Neuausrichtung der Arbeitsmarktpolitik geht mit tiefgreifenden Veränderungen in der Arbeitsmarktstatistik und der Arbeitsmarktberichterstattung einher. Damit verlieren die etablierten und auch der breiten Öffentlichkeit geläufigen Kennziffern des Arbeitsmarktgeschehens (z.B. Arbeitslosenzahlen und -quoten) an Erklärungskraft. Neue, erklärungsbedürftige Daten und Kennziffern treten ihnen zur Seite und werden für die Steuerung und das Controlling der BA, der Arbeitsgemeinschaften von Kommunen und Arbeitsagenturen nach $₫ 44 \mathrm{~b}$ und der anderen Akteure der Arbeitsmarktpolitik bedeutsam.

Obwohl die BA aufgrund der Experimentierklausel zum SGB II erstmals seit ihrer Entstehung nur noch für einen Teil der Arbeitslosen zuständig ist, hat die BA-Statistik nach den $\$ \$ 280$ ff. SGB III und $\$ 53$ SGB II weiterhin allein den gesetzlichen Auftrag, unter Einschluss aller Daten aus dem Leistungsbereich des SGB II eine umfassende Arbeitsmarktstatistik über Arbeitslosigkeit, Leistungsbezug und Förderung zu erstellen. Ab Berichtsmonat September 2005 weist die BA-Statistik erstmals seit Bestehen des neuen Leistungssystems SGB II den Bestand an Arbeitslosen wieder vollständig aus, d.h. einschließlich der arbeitslosen SGB-II-Bezieher|innen in den Optionskommunen, die zum Teil noch geschätzt werden.

\section{Mengeneffekte der Zusammenlegung von Arbeitslosen-und Sozialhilfe}

In einem ersten Sonderbericht zum "Übergang von der Arbeitslosen- und Sozialhilfe zur Grundsicherung für Arbeitslose« vom August 2005 hat die BA auf Basis einer Revision der monatlich aktuell veröffentlichten Daten der Monate Januar bis März² 2005 dargestellt, welche Wirkungen die gesetzlichen Änderungen auf den Leistungsbezug und die Arbeitslosigkeit hatten. ${ }^{3}$ Inzwischen liegen revidierte Daten bis einschließlich Juli 2005 vor (Bundesagentur für Arbeit 2005), außerdem werden monatlich aktuelle Daten veröffentlicht, die allerdings immer noch teilweise auf Schätzungen beruhen.

Durch den Umstieg auf die Grundsicherung für Arbeitsuchende im Januar 2005 stieg die Zahl der Leistungsempfänger|innen gegenüber der Situation vor dem Systemwechsel um rund 580.00o Personen an (vgl. Tabelle I): Im Dezember 2004 erhielten 3,92 Millionen Personen im erwerbsfähigen Alter zwischen Is und 65 Jahren Sozial- oder Arbeitslosenhilfe (bereinigt um geschätzte Doppelzählungen von Arbeitslosenhilfebeziehenden mit aufstockender Sozialhilfe). Darunter waren 2,26 Millionen Arbeitslosenhilfe- und I,87 Millionen

2 Die Revision ist aus zwei Gründen erforderlich: Zum einen werden für einen Teil der Arbeitsuchenden Leistungsanträge erst nach dem Zeitraum bewilligt, für den sie beantragt wurden, zum anderen ist die Datenlage in einigen Kreisen noch unzureichend, so dass für die aktuelle Berichterstattung ein Schätzverfahren genutzt wird. Die Revision der aktuellen statistischen Daten erfolgt regelmäßig nach Ablauf von drei Monaten Wartezeit.

3 Vor der Gesetzesänderung wurden von der BA die Statistiken zur Arbeitslosenhilfe und vom Statistischen Bundesamt die Statistiken zur Sozialhilfe veröffentlicht. 
Sozialhilfeempfänger|innen (jeweils inklusive Arbeitslosenhilfempfänger|innen mit aufstockender Sozialhilfe). Nach Umstieg auf das neue System erhielten im Januar 2005 4,5 Millionen erwerbsfähige Hilfebedürftige das neue Alg II.

\section{Tabelle I: Mengeneffekte des Systemwechsels}

\begin{tabular}{ll}
\hline Ende Dezember 2004 & Januar 2005 \\
\hline 3,92 Mio. Arbeitslosenhilfe- und Sozialhilfeempfänger|innen & $\begin{array}{l}4,50 \text { Mio. Arbeitslosengeld II- } \\
\text { im Alter zwischen } 15 \text { und } 65 \text { Jahren }\end{array}$ \\
Empfänger|innen
\end{tabular}

Quelle: Bundesagentur für Arbeit

Nicht alle früheren Arbeitslosenhilfeempfänger|innen erhielten das Alg II, ${ }^{4}$ da die Anspruchsvoraussetzungen beim Alg II enger sind (z.B. Anrechnung von Einkommen von Angehörigen der Bedarfsgemeinschaft). Andererseits wurden in der Arbeitslosenhilfestatistik nur die betroffene Person selbst, nicht aber ihre Angehörigen erfasst, während in der Grundsicherung für Arbeitsuchende alle Mitglieder der Bedarfsgemeinschaft einen Anspruch auf Alg II haben, wenn sie erwerbsfähig sind (bzw. auf Sozialgeld, wenn sie nicht erwerbsfähig sind), und deshalb statistisch als Leistungsempfänger|innen erfasst werden. ${ }^{5}$ Eine andere Erklärung für den Anstieg könnte nach Aussagen der BA darin liegen, dass die neue Grundsicherung für Arbeitsuchende insbesondere von Hilfebedürftigen mit niedrigem Einkommen stärker in Anspruch genommen wird als die alte Sozialhilfe. ${ }^{6}$

Im Laufe des Jahres stiegen die Empfängerzahlen von Alg II weiter an. Nach revidierten Daten der BA nahm die Zahl der hilfebedürftigen Personen von Januar bis Juli 2005 um zwölf Prozent zu. Im Juli 2005 erhielten 9,2 Prozent der Menschen im Alter zwischen I5 und 65 Jahren Alg II. Der Anteil der erwerbsfähigen Hilfebedürftigen an der Bevölkerung im Erwerbsalter (Hilfequote) ist in Ostdeutschland mit 15,6 Prozent mehr als doppelt so groß wie in Westdeutschland (7,4 Prozent). Hohe Hilfequoten weisen neben den ostdeutschen Bundesländern die Stadtstaaten auf: Berlin mit 17,I Prozent, Bremen mit 15,8 Prozent und Hamburg mit II,7 Prozent.

Die 5,06 Millionen erwerbsfähigen Hilfebedürftigen im Juli 2005 setzten sich zu 5I Prozent aus Männern und zu 49 Prozent aus Frauen zusammen. Betrachtet man die Hilfequo-

4 Die BA schätzt diesen Anteil auf sieben Prozent der Arbeitslosenhilfeempfänger|innen vom Dezember 2004.

5 Auf Basis der Daten aus der Einkommens- und Verbrauchsstichprobe 2003 des Statistischen Bundesamtes schätzt die BA, dass aufgrund dieses Zusammenhanges etwa 600.000 Personen, die im alten System nicht erfasst waren, eine Anspruchsberechtigung für Alg II besitzen.

6 Diese Annahme geht auf eine Studie von Wilde/Kubis (2005) zurück, wonach rund 43 Prozent der bedürftigen Haushalte die Sozialhilfe nicht in Anspruch genommen haben, weil Ansprüche gering waren, die Sozialhilfe stigmatisierend wirkte und der finanzielle Engpass eher kurzfristig war. 
ten, so ist die Betroffenheit von Männern (9,3 Prozent) und Frauen (9,I Prozent) in etwa gleich groß. Überdurchschnittlich hoch sind die Hilfequoten der Alleinerziehenden mit 29,9 Prozent, die der Ausländer|innen mit I6,6 Prozent und die der I5- bis unter 25-jährigen Personen mit II,I Prozent. Während die Betroffenheit in der Altersgruppe der I5- bis unter 25-Jährigen bei Frauen etwas größer ist als bei Männern, sind die Männer in der Altersgruppe der 50- bis unter 65-Jährigen öfter als Frauen auf Alg II angewiesen.

\section{Ausmaß der Arbeitslosigkeit nach dem Systemwandel}

In der öffentlichen Wahrnehmung wird unfreiwillige Beschäftigungslosigkeit bislang mit dem sozialstatistischen Konzept der Arbeitslosigkeit gleichgesetzt, das den prozessproduzierten Daten der BA zu Grunde liegt. Arbeitslos im sozialrechtlichen Sinne sind Arbeitsuchende von I5 bis unter 65 Jahren, die nicht in einem Beschäftigungsverhältnis stehen (Beschäftigungslosigkeit) oder weniger als I5 Stunden wöchentlich arbeiten, aktiv eine versicherungspflichtige Beschäftigung suchen (Eigenbemühungen) und der Vermittlung durch die BA sofort zur Verfügung stehen (Verfügbarkeit). Als arbeitslos registriert werden also nur Personen, auf die jede dieser Bedingungen zutrifft und die sich persönlich bei der Agentur für Arbeit gemeldet haben. Während geringfügig Erwerbstätige gleichzeitig arbeitslos gemeldet sein können, werden aufgrund von Sonderregelungen einige beschäftigungslose Personengruppen grundsätzlich nicht als Arbeitslose gezählt: zum Beispiel Personen, die nicht arbeiten können oder dürfen, sowie Ältere ab 58 Jahren.

Die neuere Arbeitsmarktgesetzgebung hatte auf dieses sozialstatistische Konstrukt der Arbeitslosigkeit gegenläufige Auswirkungen. Einerseits wird seit Anfang 2005 ein größerer Teil der erwerbsfähigen Bezieher|innen von Leistungen nach SGB II arbeitslos gemeldet, als dies früher in der Sozialhilfe der Fall war. Andererseits gelten nun - anders als früher - Leistungsbezieher|innen in Maßnahmen aktiver Arbeitsmarktpolitik auch dann nicht mehr als arbeitslos, wenn diese Maßnahmen - wie etwa Trainings- und Eingliederungsmaßnahmen oder Arbeitsgelegenheiten (»Ein-Euro-Jobs«) - nicht als Beschäftigung zählen und die Teilnehmer|innen der Vermittlung weiter zur Verfügung stehen ( $\$$ I6 Abs. 2 SGB III). Aufgrund dieser Änderungen sind Zahlen des Jahres 2005 über die registrierte Arbeitslosigkeit mit Angaben für die Vorjahre nicht vergleichbar.

Durch die Zusammenlegung von Arbeitslosen- und Sozialhilfe ist die Arbeitslosigkeit in den ersten Monaten des Jahres 2005 deutlich gestiegen. Dies hat vor allem zwei Gründe: Zum einen werden ehemalige Sozialhilfeempfänger|innen, die nun Alg II beziehen, in stärkerem Maße als Arbeitslose erfasst, ${ }^{7}$ zum anderen müssen sich nach SGB II nun auch die erwerbsfähigen Angehörigen von ehemaligen Arbeitslosenhilfebezieher|innen durch den Einsatz ihrer eigenen Arbeitskraft um die Reduzierung oder Überwindung der Hilfebedürftigkeit bemühen und werden als arbeitslos registriert, wenn ihnen eine Arbeit zugemutet werden kann. Dieser Effekt konzentriert sich nach Angaben der BA zu 68 Prozent

7 Nach der Sozialhilfeempfängerstatistik gab es Ende 2004937.000 Sozialhilfeempfänger|innen im erwerbsfähigen Alter, die nicht als arbeitslos registriert waren. 
auf Frauen. Diese Erhöhung der Arbeitslosigkeit der Frauen im Kontext der Systemumstellung steht potenziell ein arbeitslosigkeitsvermindernder Effekt entgegen, der durch den Übergang in den Nichtleistungsempfang (und damit zusammen hängenden Verzicht auf Arbeitslosmeldung) aufgrund der Anrechnung der Erwerbseinkommen von Angehörigen und des vorhandenen Vermögens zustande kommt. ${ }^{8}$ Tabelle 2 zeigt einen Vergleich der registrierten Arbeitslosigkeit im Dezember 2005 und Dezember 2004:

Tabelle 2: Arbeitslose im Dezember 2005 und 2004

\begin{tabular}{lrrrr}
\hline Region & \multicolumn{2}{c}{ Arbeitslose } & $\begin{array}{c}\text { Veränderung } \\
\text { in Prozent }\end{array}$ & $\begin{array}{c}\text { Arbeitslosenquote } \\
12 / 2005^{1}\end{array}$ \\
& $\begin{array}{c}\text { Dezember } \\
\text { 2004 }\end{array}$ & $\begin{array}{c}\text { Dezember } \\
\mathbf{2 0 0 5}\end{array}$ & \\
\hline Frauen & & & & \\
\hline Westdeutschland (ohne Berlin) & 1.216 .482 & 1.464 .498 & $+20,4$ & 10,5 \\
\hline Ostdeutschland (inklusive Berlin) & 751.218 & 707.036 & $-5,9$ & 18,5 \\
\hline Bundesrepublik insgesamt & 1.967 .700 & 2.171 .534 & $+10,4$ & 12,2 \\
\hline Männer & & & & \\
\hline Westdeutschland (ohne Berlin) & 1.645 .622 & 1.655 .811 & $+0,6$ & 10,7 \\
\hline Ostdeutschland (inklusive Berlin) & 851.094 & 778.717 & $-8,5$ & 19,5 \\
\hline Bundesrepublik insgesamt & 2.496 .716 & 2.434 .528 & $-2,5$ & 12,5 \\
\hline Insgesamt & & & & \\
\hline Westdeutschland (ohne Berlin) & 2.862 .104 & 3.120 .309 & $+9,0$ & 10,6 \\
\hline Ostdeutschland (inklusive Berlin) & 1.602 .312 & 1.485 .753 & $-7,3$ & 19,0 \\
\hline Bundesrepublik insgesamt & 4.464 .416 & 4.606 .062 & $+3,2$ & 12,4 \\
\hline
\end{tabular}

I Bezogen auf die abhängigen zivilen Erwerbspersonen.

Quelle: Bundesagentur für Arbeit, Berechnungen des Bremer Instituts für Arbeitsmarktforschung und Jugendberufshilfe

Der Vergleich der Arbeitslosenzahlen von Dezember 2005 und 2004 zeigt, dass die Veränderung der Arbeitslosigkeit (die zu einem großen Teil auf die unterschiedliche Erfassung vor und nach dem Systemwechsel zurück zu führen ist) in Ost- und Westdeutschland sehr unterschiedlich ist:

8 Somit bleibt auch durch die Umstellung auf das neue System das Problem ungelöst, dass durch die registrierte Arbeitslosigkeit der tatsächliche Umfang der Erwerbsarbeit suchenden Personen unvollständig abgebildet wird. 
- In Westdeutschland und in allen westdeutschen Bundesländern mit Ausnahme von Bayern, wo es keine Veränderungen in der Gesamtzahl der Arbeitslosen gab, ist die Arbeitslosigkeit teilweise stark angestiegen. Die Veränderungsraten liegen zwischen +3,6 Prozent in Rheinland-Pfalz und +20,o Prozent in Hamburg. Während bei den Männern das Bild auf Länderebene uneinheitlich ist (sowohl Rückgang als auch Zunahme der Arbeitslosigkeit), stieg die Frauenarbeitslosigkeit in allen westdeutschen Bundesländern stark an - zwischen +9 Prozent in Bayern und +33,8 Prozent in Hamburg.

- In Ostdeutschland und in ausnahmslos allen ostdeutschen Bundesländern ist die Arbeitslosigkeit hingegen sowohl bei den Männern als auch bei den Frauen zurück gegangen. Die Veränderungsraten liegen bei den Männern zwischen -IO,I Prozent in Thüringen und -I6 Prozent in Mecklenburg-Vorpommern und bei den Frauen zwischen -fünf Prozent in Sachsen und -II,8 Prozent in Mecklenburg-Vorpommern.

- Westdeutsche Frauen sind nach der Systemumstellung in deutlich stärkerem Ausmaß als bislang als Arbeitslose registriert. Ostdeutsche Frauen gelten hingegen seltener als vor der Neuregelung als arbeitslos, was möglicherweise ${ }^{9}$ an den engeren Zugangsbedingungen zur Leistung Alg II (gegenüber dem Zugang zur früheren Arbeitslosenhilfe) liegt; Frauen in Ostdeutschland erhielten nämlich häufiger Arbeitslosen- und seltener Sozialhilfe als westdeutsche Frauen.

\section{Umbau und Ausweitung der Arbeitsmarktstatistik}

Der Streit um die Wirkungen der "Hartz-Gesetze« wird in den nächsten Jahren auf der Grundlage einer unsicheren und im Umbau begriffenen Arbeitsmarkt- und Sozialstatistik geführt werden, die außerhalb eines engen Kreises von Fachleuten in hohem Maße erläuterungsbedürftig bleiben dürfte. Wichtiger als die gemeldete Arbeitslosigkeit könnte die »breite« Arbeitslosigkeit (vgl. Oschmiansky/Oschmiansky 2003) werden, d.h. ein gegen sozialrechtliche Änderungen stabiles Darstellungskonzept für die gesamtwirtschaftliche Beschäftigungslücke, in dem sich Verschiebungen zwischen Arbeitslosigkeit, Erwerbslosigkeit und stiller Reserve abbilden lassen. Darüber hinaus werden Bewegungsdaten, d.h. Zugänge in und Abgänge aus Arbeitslosigkeit, Übergänge zwischen verschiedenen Erwerbsstatus und zwischen den Leistungssystemen - nach SGB III, SGB II und SGB XII -, an Bedeutung gewinnen, und die Bewertung neuer Kennzahlen der Arbeitsmarktdynamik wird den Gegenstand öffentlicher Kontroversen bilden.

Für international vergleichende Statistiken, vor allem zur Berechnung der EU-standardisierten Erwerbslosenquoten, wird in Bevölkerungsumfragen - Mikrozensus (MZ) und EU-Arbeitskräfteerhebung (EU-AKE) - bereits seit längerem die im Labour-Force-Konzept der International Labour Organisation (ILO) definierte Erwerbslosigkeit erhoben. Dieses Konzept ist unempfindlich gegen sozialrechtliche Änderungen. Erwerbslos sind nach dem ILO-Konzept Personen ab is Jahren (ohne obere Altersgrenze), die zum Zeitpunkt der Er- 
hebung (Berichtswoche) nicht erwerbstätig, jedoch gegenwärtig (in den zwei auf die Erhebung folgenden Wochen) für Erwerbsarbeit verfügbar waren, und die sich innerhalb der letzten vier Wochen aktiv um eine Stelle bemühten bzw. die einen Arbeitsplatz gefunden, aber noch nicht angetreten hatten. Erfasst werden also auch Arbeitsuchende, die bei den Agenturen für Arbeit nicht gemeldet sind. Andererseits sind die statistischen Kriterien für Verfügbarkeit und Eigenbemühungen strenger definiert, und Erwerbstätigkeit (ab der ersten geleisteten Stunde) schließt Erwerbslosigkeit aus. Seit Januar 2005 weist das Statistische Bundesamt parallel zur BA und zusätzlich zum MZ eine monatliche Erwerbslosenquote auf Basis der Telefonerhebung Arbeitsmarkt in Deutschland (AiD) aus, der dieses Konzept zugrunde liegt.

Auch wenn sich die Messkonzepte "Arbeitslosigkeit« und »Erwerbslosigkeit« teilweise überschneiden, erfassen sie zusammen nur einen Ausschnitt aus dem Personenkreis, der seinen Erwerbswunsch nicht realisieren kann. Die stille Reserve bildet den Teil des Arbeitskräfteangebots, der weder die sozialrechtlichen noch die erwerbsstatistischen Anforderungen aktiver Arbeitsuche und kurzfristiger Verfügbarkeit erfüllt. Zu ihr zählen: Beschäftigungslose, welche die Agentur für Arbeit nicht einschalten, die Arbeitsuche entmutigt aufgegeben haben, nicht kurzfristig verfügbar sind bzw. an Maßnahmen der Arbeitsförderung teilnehmen; Nichterwerbstätige, die in absehbarer Zeit (z. B. nach einer Familienphase) eine Arbeit aufnehmen möchten; sowie Ältere, die aus Arbeitsmarktgründen vorzeitig aus dem Erwerbsleben ausgeschieden sind. Gemeinsam ist diesen Gruppen, dass sie bei einer anderen Arbeitsmarktsituation wahrscheinlich erwerbstätig bzw. erwerbslos wären.

Übergänge in die stille Reserve und aus der stillen Reserve lassen sich jedoch nicht beobachten, solange diese wie derzeit beim IAB nur als gesamtwirtschaftliche Aggregatgröße geschätzt wird. Um Bewegungen zwischen der stillen Reserve, dem Beschäftigungssystem und der Arbeitslosigkeit bzw. Erwerbslosigkeit zu verfolgen, müsste diese vielmehr auch nach einem Mikroansatz anhand von Umfragedaten zur Erwerbsabsicht direkt berechnet werden (vgl. Holst 2000, Seifert 2003). Eine solche Abschätzung der stillen Reserve anhand von Mikrodaten ist nach dem Erhebungsdesign ab 2005 sowohl mit dem Mikrozensus als auch mit der Befragung "Arbeitsmarkt in Deutschland" möglich.

Die Einführung einer einheitlichen Kundennummer bei der BA ab 2000 und der Umstieg auf eine neue Datenplattform ermöglichen seit einigen Jahren die Verknüpfung verschiedener Informationen zu integrierten Längsschnitt-Datensätzen, die individuelle Verlaufsinformationen enthalten (vgl. Kohlmann 2004). Die wichtigste dieser Dateien, die Integrierte Erwerbsbiografie (IEB), enthält auf Personenebene Angaben im Längsschnitt über Zeiten der Beschäftigung, der Arbeitsuche, des Leistungsbezugs und der Maßnahmeteilnahme. Mit diesen Daten, die relativ zeitnah zur Verfügung stehen, ${ }^{\text {Io }}$ eröffnen sich sowohl für Monitoring und Evaluation einzelner Maßnahmen wie für die Beobachtung des Arbeitsmarktgeschehens ganz neue Analysemöglichkeiten. Darüber hinaus lassen sich Daten der

IO Eine Vollversion der IEB steht frühestens I8 Monate nach Ablauf eines Beobachtungsjahres zur Verfügung. Mit einer Vorversion, die einen Füllgrad von etwa 95 Prozent aufweist, kann jedoch bereits im Juli bzw. August nach Ablauf des Beobachtungsjahres gerechnet werden. 
Beschäftigten- und Leistungsempfänger-Historik mit Daten des IAB-Betriebspanels zu "Linked-Employer-Employee-Daten« zusammenführen, die es ermöglichen, Daten über die individuelle Erwerbsbeteiligung im Zusammenhang mit der Stellung im Betrieb zu analysieren (vgl. Alda et al. 2004b).

Diese integrierten Datensätze bilden die Grundlage für alle Vorhaben der Wirkungsforschung zu den »Hartz-Gesetzen« und des Data Warehouse, das sich in der BA im Aufbau befindet. Sie sind zugleich über das Forschungsdatenzentrum der BA im IAB teils als anonymisierte Stichproben (Scientific Use Files), teils über kontrollierte Datenfernverarbeitung und Gastarbeitsplätze unter Wahrung des Datenschutzes für externe Wissenschaftler|innen zugänglich und dürften künftig für die Beobachtung des Arbeitsmarktgeschehens breit genutzt werden.

Die verbesserte Verfügbarkeit erwerbsbiografischer Informationen und Beobachtungen zu Wechseln des Erwerbsstatus beginnt auch die Praxis der BA zu verändern. Die Projekte Biodaten und TrEffer ${ }^{\text {II }}$ sollen den Fachkräften der Agenturen statistisch-ökonometrische Auswertungen dieser Daten als Entscheidungsgrundlage bei der Zuweisung von Maßnahmen zur Verfügung stellen (vgl. Brinkmann/Stephan 2005).

\section{Konzept und Stand der Wirkungsforschung}

Die Umsetzung der »Hartz-Gesetze« ist Gegenstand zahlreicher Vorhaben der Wirkungsforschung. Aufgrund des erheblichen Mitteleinsatzes und des privilegierten Daten- und Feldzugangs wird die Wirkungsforschung mit parlamentarischem bzw. gesetzlichem Auftrag die Arbeitsmarktforschung der nächsten Jahre prägen. Dabei sind nach dem Auftrag drei Forschungsstränge zu unterscheiden: die Ressortforschung des Bundesministeriums für Wirtschaft und Arbeit (BMWA) bzw. Arbeit und Soziales (BMAS) zur Umsetzung von Hartz I bis III, die Wirkungsforschung des BMWA (BMAS) zur Experimentierklausel in Hartz IV unter Beteiligung der Bundesländer, sowie die Wirkungsforschung des IAB. ${ }^{12}$ Die Evaluationskonzepte hierfür beruhen wesentlich auf den neuen statistischen Möglichkeiten der beim IAB geschaffenen integrierten Datenbasis für die Registerdaten der BA.

Die Evaluationsfragen des Bundestages werden in drei Arbeitspaketen bearbeitet: »Wirksamkeit der Instrumente" (Arbeitspaket I), „Organisatorischer Umbau der Bundesagentur für Arbeit« (Arbeitspaket 2), »Akzeptanz der Bundesanstalt für Arbeit« (Arbeitspaket 3). Zwei weitere Arbeitspakete, die »Administrative Unterstützung bei der Evaluation« (Koordination, Arbeitspaket 4) und »Datenbereitstellung" (Arbeitspaket 5), sollen unterstützend wirken.

II TrEffer steht für »Treatment Effects and Predictions«; Vorbild ist das Schweizer Verfahren Statistically Assisted Programme Selection (SAPS). Im Mittelpunkt dieser Verfahren steht die erwartete Dauer des "Kundenkontakts«.

I2 Neben diesen politisch mandatierten Vorhaben sind eine Reihe eigenständiger Untersuchungsvorhaben aus Mitteln der Forschungsförderung (auch bei der Hans-Böckler-Stiftung) bekannt, und weitere sind zu erwarten. 
Inzwischen liegen die ersten sechs Berichte aus dem Arbeitspaket I vor, die sechs unterschiedliche Themen (Module) im Rahmen der mit Hartz I bis III verbundenen Veränderungen behandeln:

- Wissenschaftszentrum Berlin für Sozialforschung (WZB)/Institut für angewandte Sozialwissenschaft (infas): Evaluation der Maßnahmen zur Umsetzung der Vorschläge der Hartz-Kommission. Modul Ia: Neuausrichtung der Vermittlungsprozesse, Berlin und Bonn, Juni 2005

- Forschungsinstitut zur Zukunft der Arbeit (IZA)/Institut für angewandte Sozialwissenschaft (infas)/Deutsches Institut für Wirtschaftsforschung (DIW): Evaluation der Maßnahmen zur Umsetzung der Vorschläge der Hartz-Kommission: Modul Ib: Förderung beruflicher Weiterbildung und Transferleistungen, Bonn und Berlin, Juni 2005

- COMPASS Gesellschaft für Informationsmanagement und Projektentwicklung/Institut für Medienforschung und Urbanistik (IMU)/Institut für Sozialökonomische Strukturanalysen (SÖSTRA)/Progress-Institut für Wirtschaftsforschung (PIW)/Universität Hamburg: Evaluation der Maßnahmen zur Umsetzung der Vorschläge der Hartz-Kommission: Modul Ic: Arbeitsbeschaffungsmaßnahmen, Berlin, Bremen und Hamburg, Juni 2005

- Zentrum für Europäische Wirtschaftsforschung (ZEW)/Institut für Arbeitsmarkt- und Berufsforschung (IAB)/Institut Arbeit und Technik (IAT): Evaluation der Maßnahmen zur Umsetzung der Vorschläge der Hartz-Kommission: Modul Id: Eingliederungszuschüsse und Entgeltsicherung, Nürnberg, Gelsenkirchen und Mannheim, Juni 2005

- Institut für Arbeitsmarkt- und Berufsforschung (IAB)/Deutsches Institut für Wirtschaftsforschung (DIW)/sinus Gesellschaft für Sozialforschung und Marktforschung/ Alexander Kritikos (Gesellschaft für Arbeitsmarktaktivierung GfA)/Institut für angewandte Sozialwissenschaft (infas): Evaluation der Maßnahmen zur Umsetzung der Vorschläge der Hartz-Kommission: Modul re: Existenzgründungen, Berlin, Bonn, München und Nürnberg, Juni 2005

- Rheinisch-Westfälisches Institut für Wirtschaftsforschung (RWI)/Institut für Sozialforschung und Gesellschaftspolitik (ISG): Evaluation der Umsetzung der Vorschläge der Hartz-Kommission: Modul If: Verbesserung der beschäftigungspolitischen Rahmenbedingungen und Makrowirkungen der aktiven Arbeitsmarktpolitik, Essen und Köln, Juni 2005

Die Umsetzung des SGB II und die Kooperation zwischen Agenturen für Arbeit und Kommunen wird in diesen Evaluationen nur am Rande, nämlich als Rahmenbedingung für Leistungsprozesse nach SGB III, berücksichtigt: So ist in den Modulen Ia bis Id die Anwendung der jeweiligen Instrumente durch die örtlichen Arbeitsgemeinschaften zwischen Arbeitsagenturen und Kommunen in die Untersuchung einbezogen. Dagegen werden weder die Veränderungen bei den Lohnersatzleistungen nach SGB III - also die Zusammenführung von Unterhaltsgeld (Uhg) und Arbeitslosengeld (Alg) und die Verkürzung der Anspruchsdauer beim Alg - noch das Alg II bzw. das SGB II als besonderes Leistungssystem 
untersucht. Die Optionskommunen als alleinige Träger des SGB II sind ausdrücklich aus der Beobachtung ausgeschlossen.

Das Institut für angewandte Sozialwissenschaft (infas, Bonn) bearbeitet die Untersuchung der Akzeptanz der BA bei Arbeitgeber|inne|n, Arbeitnehmer|inne|n und in der Bevölkerung (Arbeitspaket 3); hierzu werden eine Stichprobe der Wohnbevölkerung ab I8 Jahren, eine zweite Stichprobe aus Beschäftigten und Arbeitslosen sowie eine Betriebsstichprobe zu drei Zeitpunkten im Frühjahr 2004, 2005 und 2006 befragt. Die Studie, in der die neuen Organisationsstrukturen, die Implementierung des neuen Steuerungsmodells in der BA, das Verhältnis von Geschäftsleitung und Selbstverwaltung, die Rolle der Beschäftigten im Umbau und die Bedeutung neuer IT-Verfahren für die Arbeitsabläufe untersucht werden, wird vom Institut für Sozialforschung und Sozialwirtschaft (iso-Institut, Saarbrücken) und der Organisationsberatung Peter Ochs (Saarbrücken) bearbeitet.

Der $\$ 6 c$ SGB II sieht für die Experimentierklausel zur Grundsicherung und Vermittlung Arbeitsuchender in kommunaler Trägerschaft (kommunale Option) eine eigene Evaluierung vor, für die das BMWA (BMAS) in Zusammenarbeit mit den Ländern verantwortlich ist (vgl. BMWA 2004). Zu diesem Bereich der Wirkungsforschung hat sich im Januar 2005 nach einer schriftlichen Abfrage bei Ländern, kommunalen Spitzenverbänden, Arbeitgeberverbänden und Deutschem Gewerkschaftsbund (DGB) ein Arbeitskreis Evaluation konstituiert. Bisher sind die ersten beiden von vier definierten Untersuchungsfeldern vergeben worden: Deskriptive Analyse und Matching (Feld I) und Implementations- und GovernanceAnalyse (Feld 2). Ausschreibungen zur Wirkungs- und Effizienzanalyse (Feld 3) sowie zu Makroanalyse und Benchmarking (Feld 4) sind in Vorbereitung. Die Untersuchungen umfassen die ersten drei Jahre der Umsetzung des SGB II, also den Zeitraum von Mitte 2005 bis Mitte 2008. \$ 6c SGB II verlangt zum 31. Dezember 2008 einen Bericht des BMWA an Bundestag und Bundesrat. Im Zentrum dieser Forschungsvorhaben steht ein Leistungsvergleich der beiden grundlegenden Trägermodelle, d.h. der Arbeitsgemeinschaften nach $\$ 44 \mathrm{~b}$ SGB II und der kommunalen Trägerschaft nach $\$$ 6a SGB II.

Das Ministerium für Wirtschaft und Arbeit (MWA) bzw. für Arbeit, Gesundheit und Soziales (MAGS) des Landes NRW bereitet parallel ein landesweites Evaluationsvorhaben zur Wahrnehmung der kommunalen Option des SGB II vor, und auch Hessen plant eine SGB-II-Evaluation auf Landesebene. ${ }^{13}$

Grundsätzlich und längerfristig ist nach $\$ 282$ SGB III und $\$ 55$ SGB II das IAB für die Wirkungsforschung in beiden Regelungsbereichen der Arbeitsförderung zuständig. Für die allgemeine Wirkungsforschung im Bereich der Grundsicherung für Arbeitsuchende hat das IAB die Forschungsbereiche "SGB II: Soziale Sicherung und Partizipation", "SGB II: Effektivität und Effizienz von Leistungen " und "SGB II: Strukturen und Bewegungen im Niedrigeinkommensbereich" neu eingerichtet. Auf mittlere und lange Sicht sieht das Forschungsprogramm (zusammenfassend vgl. Beckmann et al. 2004) Implementationsstudien,

I3 Ob auch andere Bundesländer eine parallele Evaluation auf Landesebene planen, ist den Autor|inn|en nicht bekannt. 
Analysen zum Instrumenteneinsatz auf der Individualebene, Forschungen zu den Bedarfsgemeinschaften nach SGB II und ihrem Haushaltskontext, Untersuchungen auf der Betriebsebene und zur Reduzierung der Arbeitslosigkeit auf regionaler Ebene sowie zu den gesamtwirtschaftlichen Effekten des neuen Leistungssystems vor.

\section{Probleme und Defizite der Wirkungsforschung}

Trotz (oder wegen) ihres Umfangs wird die Wirkungsforschung als Grundlage einer kritischen Begleitung und Meinungsbildung zu den »Hartz-Gesetzen« nicht ausreichen. Anhand der Ausschreibungen und der vorliegenden Evaluationsdesigns lassen sich eine Reihe von Problemen absehen.

Handlungsbedarf ergibt sich zunächst bereits aus der großen Zahl verschiedener Forschungsvorhaben, deren Ergebnisse nur einen kleinen Kreis von Expert|inn|en erreichen dürften. Man müsste eine sehr naive Vorstellung vom politikberatenden Nutzen der Evaluation haben, wollte man annehmen, dass die Ergebnisse eines so umfangreichen Forschungsprogramms für sich sprechen könnten. Wie sie im öffentlichen Diskurs über die Arbeitsmarktpolitik wirken, wird vielmehr von Deutungsangeboten und Übersetzungen abhängen. Dies gilt insbesondere auch für die parlamentarische Auseinandersetzung über die zusammenfassenden Berichte, welche die Bundesregierung Bundestag und Bundesrat zuleiten wird. Die Meinungsbildung über die Wirkungen der »Hartz-Gesetze« kann nur an demokratischer Qualität gewinnen, wenn sich neben den Auftraggeber|inne|n und Evaluator|inn|en auch andere Wissenschaftler|innen am Transfer und an der Erörterung der Ergebnisse beteiligen.

Dass es notwendig und nützlich ist, die Wirkungsforschung ihrerseits fachöffentlich kritisch zu begleiten, ergibt sich ferner auch aus der Schneidung der einzelnen Forschungsvorhaben und aus ungelösten konzeptionellen Fragen. Die Arbeitsteilung zwischen relativ kurzfristiger Ressortforschung einerseits und dem längerfristigen Forschungsprogramm des $\mathrm{IAB}$ andererseits sowie die Trennung der Forschungsaufträge nach den Regelkreisen des SGB III und des SGB II, noch dazu mit unterschiedlichen Zeithorizonten (bis 2006 bzw. 2008), werden schwerlich ein Gesamtbild der neu ausgerichteten Arbeitsmarktpolitik entstehen lassen. Begonnen wurden fast ausschließlich Partialevaluationen einzelner Regelungen und Instrumente. Dabei ergeben sich bereits durch die Schneidung Untersuchungslücken. So wird das SGB II in der Ressortforschung des BMWA (BMAS) nur als Sonderfall für die Anwendung von Instrumenten des SGB III und in der Wirkungsforschung nach $\$ 6 \mathrm{c}$ SGB II nur unter der Fragestellung des Leistungsvergleichs zwischen rein kommunalen und gemeinsamen Trägermodellen evaluiert - eine Gesamtevaluation der Wirkungen des neuen Leistungssystems ist daher noch am ehesten vom IAB zu erwarten.

Damit die große Anzahl an Forschungsvorhaben unter großem Zeitdruck kurzfristig begonnen werden konnten, mussten projektbezogene Konsortien gebildet werden. In den beauftragten Konsortien dominieren die mikroökonometrisch ausgerichteten Ansätze der Wirtschaftsforschungsinstitute. Insbesondere die soziologische Arbeitsmarktforschung war auf die Übernahme von Aufgaben dieser Größenordnung theoretisch und auf 
die Bildung leistungsfähiger Konsortien praktisch ungenügend vorbereitet. ${ }^{14}$ Die neuen Aufträge der Wirkungsforschung mussten ohne gründliche wissenschaftliche Verständigung über Standards qualifizierter Evaluationsforschung im veränderten Feld der Arbeitsmarktpolitik begonnen werden. Ihre Ergebnisse hängen aber entscheidend von wertgebundenen Perspektiven und Annahmen ab, die sich nicht einfach aus den gesetzlichen Grundlagen ergeben, sondern die in der einen oder anderen Weise wissenschaftlich zu »rekonstruieren « sind.

Der Auftragsvergabe ist keine ausreichende Verständigung über Zieldefinitionen und Erfolgskriterien unter Beteiligung aller relevanten Akteursgruppen vorausgegangen. So fehlt der Wirkungsforschung nun nicht nur eine Programmkritik, sondern auch ein politisch definiertes und mit den relevanten gesellschaftlichen Gruppen vereinbartes Beobachtungskonzept. Unter diesen Bedingungen und aufgrund des Zeitdrucks ist die erste Aufgabe jedes Evaluationsvorhabens, die rationale Rekonstruktion und kritische Erörterung der Programmziele, in den begonnenen Vorhaben der Begleitforschung zu kurz gekommen. Die Grundannahme der "Hartz-Gesetze«, dass Arbeitslose nicht aktiv genug Stellen suchen und dass die Unterbeschäftigung durch beschleunigte Besetzung offener Stellen erheblich reduziert werden kann, wird nicht mehr kritisch hinterfragt.

Bei den Vorhaben der Wirkungsforschung zu den »Hartz-Gesetzen« handelt es sich bislang fast ausschließlich um Partialevaluationen mit dem Ziel, einzelne Regelungen und Instrumente nach ihrer Wirksamkeit zu vergleichen, nicht intendierte Folgen bei ihrer Umsetzung zu identifizieren und so Anhaltspunkte für eine optimierte Umsetzung zu gewinnen. Hier dürfte sich das Methodenverständnis der meisten Auftragnehmer|innen, die kausale Effekte einzelner Interventionen verlässlich abbilden wollen, ${ }^{15}$ mit dem Interesse der politischen Auftraggeber|innen treffen, eine grundsätzliche Erörterung der neu ausgerichteten Arbeitsmarktpolitik zu vermeiden.

Obwohl die Ausgestaltung der Lohnersatz- bzw. Lohnergänzungsleistungen erheblichen Einfluss auf das Suchverhalten am Arbeitsmarkt und damit auf Stellenbesetzungsvorgänge hat (vgl. Gangl 2002), werden diese Leistungen nur am Rande (etwa im Zusammenhang mit Sanktionen) in die Untersuchung einbezogen. Weder die Höhe des Alg II noch seine Rechtssicherheit noch die darauf bezogenen Leistungsprozesse (Antragsbearbeitung,

I4 So der Tenor der selbstkritischen Diskussionsbeiträge und des Schlussworts von Burkart Lutz auf der Fachtagung "Wirkungsforschung und Politikberatung - eine Gratwanderung? « der Deutschen Vereinigung für sozialwissenschaftliche Arbeitsmarktforschung (SAMF), der Friedrich-Ebert-Stiftung (FES) und des IAB vom 24./25. Februar 2005 in Bonn (vgl. SAMF/IAB/FES 2005).

I5 In einer der beiden Vorstudien zur Evaluation der vermittlungsorientierten Instrumente wird der Zusammenhang der einzelnen Regelungen als methodisch auszuschaltende Störgröße bezeichnet: "Diese einzelnen Reformen [...] greifen inhaltlich und in ihrer Zielsetzung ineinander und müssen daher als ein Ganzes betrachtet und evaluiert werden [...]. Jede Evaluationsstudie, die die Ermittlung des kausalen Effekts einer Maßnahme zum Ziel hat, muss grundsätzlich einen möglichst isolierten Eingriff betrachten, um zu verhindern, dass sich der Effekt mehrerer Veränderungen überlagert. Liegen mehrere Eingriffe gleichzeitig vor, so ist es Aufgabe des Studiendesigns, eine derartige isolierte Betrachtung dennoch zu gewährleisten.« (Fertig et al. 2004: I8 f.) 
Anspruchsprüfung, Zahlbarmachung) sind Gegenstand der Evaluation durch das BMWA (BMAS). ${ }^{16}$ Dies ist problematisch, weil alle Aktivitäten zur Arbeitsmarktintegration die materielle Existenzsicherung zur Voraussetzung haben, Geldleistungen und Leistungen zu Eingliederung in den verpflichtenden Eingliederungsvereinbarungen nach $₫$ is SGB II verkoppelt sind und der größte Teil der Aktivitäten von Fachkräften und Adressat|inn|en im Regelkreis des SGB II auf die Beantragung, Prüfung und Auszahlung der Geldleistungen entfällt.

Auf der Mikroebene orientieren sich die Evaluationsvorhaben bislang fast ausschließlich an einer einzigen Zieldimension, der Ausmündung aus gemeldeter Arbeitslosigkeit bzw. der kurzfristigen Eingliederung in den allgemeinen Arbeitsmarkt. Hieran soll der Erfolg vermittlungsorientierter Maßnahmen und Dienstleistungen mittels quasiexperimenteller ökonometrischer Evaluationsdesigns gemessen werden. Ob diese Beschäftigung nach ihrer Qualität, ihrer Entlohnung und ihrer Gesamtdauer dazu führt, die Zone der Unsicherheit am Arbeitsmarkt zu verlassen, wird nicht erfasst. Andere für die Bewertung der Instrumente wichtige Dimensionen wie die Qualität der Beschäftigung, der längerfristige erwerbsbiografische Kontext kurzfristiger Eingliederungs-»Erfolge«, die Einkommenssituation und die Erwerbskonstellation des Haushalts sowie die schwerer quantifizierbaren Ziele des Erhalts oder der Verbesserung individueller Beschäftigungsfähigkeit oder der sozialen Stabilisierung in gefährdeten Lebenslagen werden ebenfalls kaum thematisiert.

Da mikroökonometrische Evaluationskonzepte dominieren, werden gesamtwirtschaftliche Niveau- und Struktureffekte, etwa durch Ausweitung von Arbeitsgelegenheiten mit Mehraufwandsentschädigung, durch die Zunahme nicht standardisierter Beschäftigungsverhältnisse und nicht existenzsichernder Arbeitseinkommen, durch die - mehr oder weniger offen intendierte - arbeitsmarktpolitische Funktion des Alg II als Lohnergänzungsleistung im Niedriglohnsegment und durch die Konkurrenz verschiedener Personengruppen der Arbeitslosen und der stillen Reserve bei Stellenbesetzungen nicht ausreichend untersucht. Das IAB-Forschungsprogramm schlägt hierzu vor, ein Mikrosimulationsmodell zur Analyse von Änderungen im Erwerbsverhalten mit einem empirischen Modell des gesamtwirtschaftlichen Arbeitsangebots zu verknüpfen (vgl. Beckmann et al. 2004: 22).

Obwohl Implementationsanalysen und qualitative Untersuchungsverfahren in allen Evaluationsvorhaben vorgesehen sind, fehlt eine grundsätzliche Auseinandersetzung mit den Besonderheiten vermittlungsorientierter »Dienstleistungen am Arbeitsmarkt«, die doch den »Hartz-Gesetzen« den Namen gaben. Im Mittelpunkt stehen bislang organisatorische Fragestellungen der Leistungsträger und der Fachkräfte. Um aber zu klären, ob ein Zusammenhang zwischen bestimmten Leistungen und beobachteten Übergängen in reguläre Beschäftigung besteht und ob die Vermittlung die Beschäftigungsfähigkeit, also den Handlungsspielraum von Arbeitsuchenden bei der Stellenbesetzung erhöht, wären in stärkerem Maße Leistungsprozesse zu beobachten und wäre die Qualität dieser Dienstleistung aus der Perspektive der »Kunden«, d.h. der Arbeitsuchenden, zu bewerten. Keines der Evaluations-

I6 Das Forschungsprogramm des IAB erwähnt immerhin die Wirkungen des SGB II auf Armutsrisiko und Einkommensverteilung (Beckmann et al. 2004: I6, 20 f.). 
vorhaben orientiert sich an einem ausgewiesenen Handlungsmodell zur Rolle der Arbeitsvermittlung in Stellenbesetzungsvorgängen, zu »Kundensegmentierung « und »Profiling«, zu Beratung und Fallmanagement.

Während alle Expert|inn|en sich darüber einig sind, dass Evaluationsergebnisse um so zuverlässiger werden, je länger die beobachtete Zeitspanne ist, standen die Projekte schon durch die Beschlüsse der Legislative unter hohem Abfragezeitdruck. Das Ziel, relativ kurzfristig Ergebnisse zu liefern, geht zu Lasten der Qualität, ohne dass der absehbare politikberatende Nutzen dies rechtfertigen könnte. Denn die Realitäten des politischen Prozesses entsprechen nicht dem Modell eines rationalen Policy-Zyklus, in dem ein Programm mit einer Problemwahrnehmung beginnt und sich an Politikformulierung und Politikumsetzung eine Phase der Korrektur aufgrund von Evaluationsergebnissen anschließt. Zu keinem Zeitpunkt während des Evaluationszeitraums bis 2006 bzw. bis 2008 wird ein stabiler Endzustand der Umsetzung der »Hartz-Gesetze« erreicht sein, der mit einem vorausgegangenen Zustand »vor Hartz« (»Nullmessung «) systematisch verglichen werden könnte. So könnten politische Entscheidungen über Programmänderungen fallen, ohne dass hierzu Ergebnisse der Wirkungsforschung vorliegen. Doch zu einer Prozessanalyse, die auch Auswirkungen des politischen Prozesses und etwaiger Programmkorrekturen auf die Umsetzung beobachtet, haben die Evaluationsvorhaben nur ein sehr schwaches Mandat.

\section{Literatur}

Alda, Holger/Hauss, Friedrich/Land, Rainer/Willisch, Andreas [Alda et al. 2004a] (2004): Erwerbsverläufe und sekundärer Integrationsmodus, Ergebnisse einer empirischen Untersuchung, in: Berliner Debatte Initial, Jg. 9, H. I5, S. 70-85

Alda, Holger/Bender, Stefan/Gartner, Hermann [Alda et al. 2004b] (2004): Der Linked Employer-Employee-Datensatz aus IAB-Betriebspanel und den prozessproduzierten Daten des IAB (LIAB), Nürnberg

Beckmann, Petra/Blien, Uwe/Brinkmann, Christian/Dietrich, Hans/Feil, Michael/Koch, Susanne/Konle-Seidl, Regina/Promberger, Markus/Rudolph, Helmut/Stephan, Gesine (2004): Forschung zum SGB II aus Sicht des IAB: Die neuen Forschungsaufgaben im Überblick, IAB-Forschungsbericht, Nr. 6/2004, Nürnberg

Brinkmann, Christian/Stephan, Gesine (2005): Better Data Help a Lot - Evaluationsforschung im Aufwind, in: IAB-Forum, Jg. I, H. I, S. $38-42$

Bundesagentur für Arbeit (2005): Grundsicherung für Arbeitsuchende. Entwicklung bis Juli 2005. Bericht der Statistik der BA, Nürnberg

Bundesministerium für Wirtschaft und Arbeit [BMWA] (2004): Erarbeitung eines Konzepts zur Evaluation der Grundsicherung für Arbeitssuchende aus der Perspektive der Experimentierklausel, Schriftliche Abfrage des BMWA bei Ländern, kommunalen Spitzenverbänden sowie BDA und DGB vom Io. Dezember 2004, Berlin

Fertig, Michael/Kluve, Jochen/Schmidt, Christoph M./Apel, Helmut/Friedrich, Werner/ Hägele, Helmut (2004): Die Hartz-Gesetze zur Arbeitsmarktpolitik - Ein umfassendes Evaluationskonzept, Berlin 
Gangl, Markus (2002): Unemployment Benefits as a Search Subsidy: New Evidence on Duration and wage Effects of Unemployment Insurance, WZB Discussion Paper, Nr. FS I o2-208, Berlin

Henke, Jutta (2004): Wenn die Fürsorge den Plan macht, Hilfeplanung in rarbeitsmarktnahen und rarbeitsmarktfernen`Systemen sozialer Sicherung, Hausarbeit am Fachbereich Sozialwesen der Universität GHK Kassel

Holst, Elke (200o): Die stille Reserve am Arbeitsmarkt: Größe, Zusammensetzung, Verhalten, Berlin

Klammer, Ute/Tillmann, Katja (2002): Flexicurity: Soziale Sicherung und Flexibilisierung der Arbeits- und Lebensverhältnisse. Forschungsprojekt im Auftrag des Ministeriums für Arbeit und Soziales, Qualifikation und Technologie des Landes Nordrhein-Westfalen, Düsseldorf

Koch, Susanne/Walwei, Ulrich (2005): Schwerpunkt Arbeitsmarktreform - Partitur im Probenraum, in: IAB Forum, Jg. I, H. I, S. 5-I3

Kohlmann, Annette (2004): Datenzugang und Datenverfügbarkeit im Forschungsdatenzentrum der Bundesagentur für Arbeit im Institut für Arbeitsmarkt- und Berufsforschung, in: Verband Deutscher Rentenversicherungsträger (VDR) (Hg.), Das Forschungsdatenzentrum der gesetzlichen Rentenversicherung (FDZ-RV) im Aufbau, Frankfurt am Main, S. $77-89$

Konle-Seidl, Regina (2005): Lessons Learned, Internationale Evaluierungsergebnisse zu Wirkungen aktiver und aktivierender Arbeitsmarktpolitik, IAB-Forschungsbericht, Nr. 9/2005, Nürnberg

Land, Rainer (2003): Ostdeutschland - fragmentierte Entwicklung, in: Berliner Debatte Initial, Jg. I4, H. 6, S. 76-95

Oschmiansky, Heidi/Oschmiansky, Frank (2003): Erwerbsformen im Wandel: Integration oder Ausgrenzung durch atypische Beschäftigung? Berlin und die Bundesrepublik Deutschland im Vergleich, WZB Discussion Paper, Nr. SP I 2003-I06, Berlin

SAMF/IAB/FES (2005): Wirkungsforschung und Politikberatung - eine Gratwanderung? Zusammenfassung der Diskussion auf der Fachtagung der Deutschen Vereinigung für Sozialwissenschaftliche Arbeitsmarktforschung (SAMF) e.V. und des IAB in Kooperation mit der Friedrich- Ebert-Stiftung (FES), Arbeitskreis Arbeit und Soziales am 24./25.2.2005 in der FES in Bonn

Seifert, Wolfgang (2003): Arbeitslosigkeit, Erwerbslosigkeit, Stille Reserve - unterschiedliche Messkonzepte zur Beschreibung der Beschäftigungslücke, in: Landesamt für Datenverarbeitung und Statistik (LDS) (Hg.): Statistische Analysen und Studien, Band I2, Düsseldorf, S. I3-I6

Wilde, Joachim/Kubis, Alexander (2005): Nichtinanspruchnahme von Sozialhilfe, in: Jahrbücher für Nationalökonomie und Statistik, Bd. 225, H. 3, S. 347-373 